- Tail bone pain

- Unintended weight loss

- History of diabetes and

hypertension

\title{
THE CASE
}

A 78-year-old man with a history of diabetes and hypertension was referred to the outpatient surgical office with a chief complaint of "tail bone pain" that had started after a fall a year earlier. The patient complained that the pain was worse when sitting and at nighttime. He also admitted to a 7-lb weight loss over the past 2 months without change in diet or appetite. He denied symptoms of incontinence, urinary retention, sharp stabbing pains in the lower extremities, night sweats, or anorexia.

The patient first visited an urgent care facility on the day after the fall because he was experiencing pain in his "tail bone" region while riding his lawn mower. A pelvic $\mathrm{x}$-ray was performed at that time and showed no coccyx fracture. He received a steroid injection in the right sacroiliac joint, which provided some relief for a month. Throughout the course of the year, he was given 6 steroid injections into his sacroiliac joint by his primary care provider (PCP) and clinicians at his local urgent care facility. One year after the fall, the patient's PCP ordered a computed tomography (CT) scan of the abdomen and pelvis, which revealed a $4.6 \times 7.5-\mathrm{cm}$ soft-tissue mass with bony destruction of the lower sacrum and coccyx that extended into the sacral and coccygeal canal (FIGURE 1).

On exam in our surgical office, the patient was found to be alert and oriented. His neurologic exam was unremarkable, with an intact motor and sensory exam and no symptoms of cauda equina syndrome. During palpation over the lower sacrum and coccyx, both tenderness and a boggy, soft mass were observed. Nerve impingement was most likely caused by the size of the mass.

\section{THE DIAGNOSIS}

Biopsy revealed a large tan-gray, gelatinous, soft-tissue mass that

\section{FIGURE 1}

\section{CT scan performed 1 year after onset} of symptoms

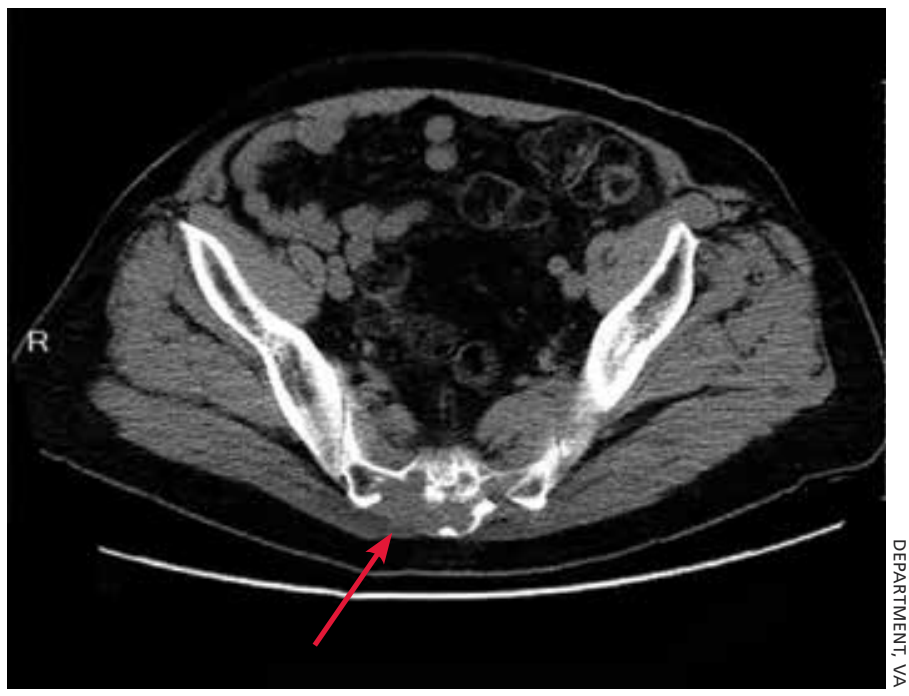

Coronal computed tomography scan of the 78-year-old patient's abdomen and pelvis showed a soft mass and local bony destruction (arrow).
Lindsey Forker, DO; Sonia Masih, DO; Scott O'Neil, MD, FACS Liberty University College of Osteopathic Medicine, Lynchburg, VA (Drs. Forker and Masih); Einstein Medical Center Montgomery, Internal Medicine Residency, East Norriton, PA (Dr. Forker) University of Toledo Medical Center, Urology Residency Program, $\mathrm{OH}$ (Dr. Masih); Martinsville Surgical Associates, SOVAH Health, VA (Dr. O’Neil)

$\rightarrow$ Imforker@gmail.com

The authors reported no potential conflict of interest relevant to this article. doi: $10.12788 /$ jfp. 0263 
FIGURE 2

\section{MRI confirmed the diagnosis}

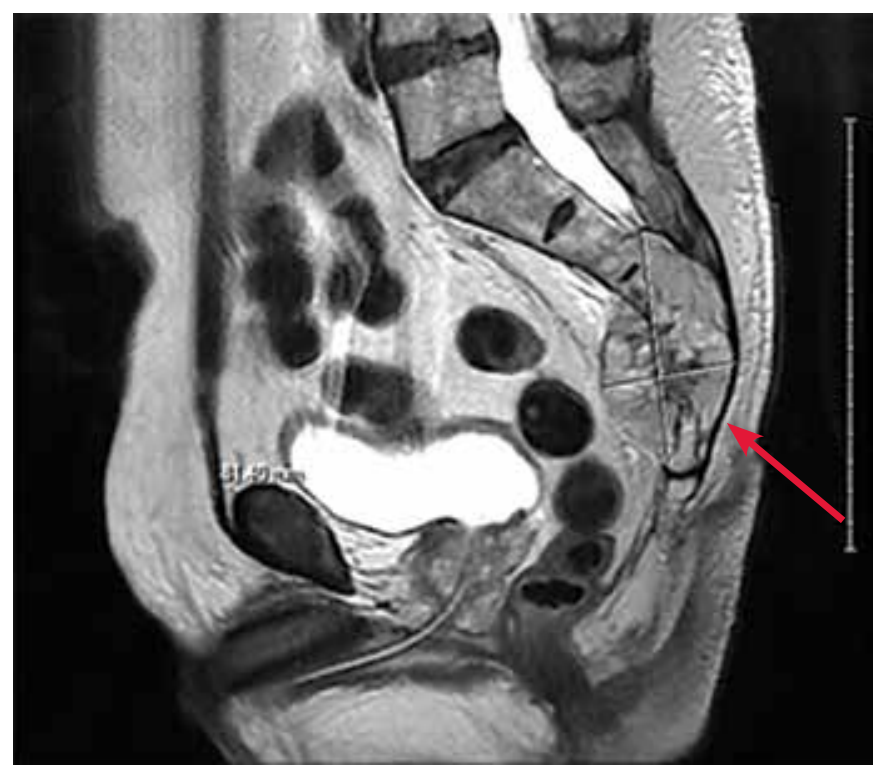

A magnetic resonance imaging scan of the patient's pelvis revealed a $4.6 \mathrm{x}$ 8.1-cm destructive, expansile sacrococcygeal tumor (arrow).

was necrotizing through the lower sacrum. The diagnosis of a sacral chordoma was confirmed with magnetic resonance imaging of the pelvis, which demonstrated a $4.6 \times 8.1-\mathrm{cm}$ destructive expansile sacrococcygeal tumor with an exophytic soft-tissue component (FIGURE 2). The tumor also involved the piriformis and gluteus maximus muscles bilaterally.

\section{DISCUSSION}

Chordomas are rare, malignant bone tumors that grow slowly and originate from embryonic remnants of the notochord. ${ }^{1}$ They are most commonly seen in the sacrococcygeal segment $(50 \%)$ but are also seen in the spheno-occipital synchondrosis (30\%-35\%) and other spinal segments such as C2 and lumbar spine. ${ }^{2}$ Chordomas are typically seen in middle-aged patients, with sacral chordomas occurring predominantly in men compared to women $(3: 1){ }^{2}$

\section{Slow to grow, slow to diagnose}

The difficulty with diagnosing sacral chordomas lies in the tendency for these tumors to grow extremely slowly, making detection challenging due to a lack of symptoms in the early clinical course. Once the tumors cause noticeable symptoms, they are usually large and extensively locally invasive. As a result, most patients experience delayed diagnosis, with an average symptom duration of 2.3 years prior to diagnosis. ${ }^{3}$

\section{Reexamining a common problem as a symptom of a rare condition}

The most commonly manifesting symptom of sacral chordomas is lower back pain that is typically dull and worse with sitting. ${ }^{3,4}$ Since lower back pain is the leading cause of disability, it is difficult to determine when back pain is simply a benign consequence of aging or muscular pain and when it is, in fact, pathologic. ${ }^{5}$ A thorough history and physical are crucial in making the distinction.

Clinical red flags include pain with neurologic symptoms (including paresthesia, urinary or bowel disturbances, and weakness in the lower limbs), pain in the lower back with or without coccyx pain that persists and gradually worsens over time, and pain that fails to resolve. ${ }^{3}$ These symptoms are collectively strong indicators of underlying sacral pathology and should warrant further investigation, including a CT and MRI of the involved area.

\section{Survival rate is improved by surgery}

The gold standard for treatment of sacral chordomas is surgical resection with adequate margins, as these tumors are both radio- and chemo-insensitive. ${ }^{6}$ It is generally accepted that achieving a wide surgical margin is the most important predictor of survival and of reducing local recurrence in patients with sacrococcygeal chordoma. ${ }^{7-9}$

The survival rate varies after a posterioronly surgical approach; some studies cite the 5-year survival rate as $100 \%$ and others state the 7 -year survival rate as $5 \% .{ }^{4}$ The wide variation is likely due to small trial size, a lack of evidence, and how invasive the disease is at the time of surgery.

The recurrence rate 5 years after surgery is approximately $20 \%{ }^{4}$ The rate of urinary and fecal incontinence after surgery using a posterior-only approach is between $20 \%$ and 
$100 \%$; some of this variation may be due to which spinal level is involved. ${ }^{4}$ If S3 is affected, there is almost always perineal anesthesia along with bowel and bladder incontinence. ${ }^{4}$

I This patient was referred to Neurosurgery and underwent resection. He recovered well from surgery but suffered from some residual urinary incontinence. The patient did not receive chemotherapy or radiation, and further work-up revealed no evidence of metastasis.

\section{THE TAKEAWAY}

The diagnosis of sacral chordoma remains challenging. A history of clinical red flags, especially persistent lower back pain with neuropathy, should prompt an aggressive investigation to rule out underlying pathology. Other signs on physical exam could include urinary or bowel disturbances, weakness in the lower limbs, saddle anesthesia, new foot drop, and/or laxity of the anal sphincter. ${ }^{5}$ Early detection and surgical intervention are crucial for these patients to experience a better prognosis and preserve maximum function.

CORRESPONDENCE

Lindsey Forker, DO, 810 Taylor Road, Collegeville, PA 19426; Imforker@gmail.com

\section{REFERENCES}

1. Zabel-du Bois A, Nikoghosyan A, Schwahofer A, et al. Intensity modulated radiotherapy in the management of sacral chordoma in primary versus recurrent disease. Radiother Oncol. 2010;97:408-412. doi: 10.1016/j.radonc.2010.10.008

2. Murphey MD, Andrews CL, Flemming DJ, et al. Primary tumors of the spine: radiologic pathologic correlation. Radiographics. 1996;1131-1158. doi: 10.1148/radiographics.16.5.8888395

3. Jeys L, Gibbins R, Evans G, et al. Sacral chordoma: a diagnosis not to be sat on? Int Orthopaedics. 2008;32:269-272. doi: 10.1007/ s00264-006-0296-3

4. Pillai S, Govender, S. Sacral chordoma: a review of literature. J Orthop. 2018;15:679-684. doi: 10.1016/j.jor.2018.04.001

5. Traeger A, Buchbinder R, Harris I, et al. Diagnosis and management of low-back pain in primary care. CMAJ. 2017;189: E1386-E1395. doi: 10.1503/cmaj.170527

6. Walcott BP, Nahed BV, Mohyeldin A, et al. Chordoma: current concepts, management, and future directions. Lancet Oncol. 2012;13:e69-76. doi: 10.1016/S1470-2045(11)70337-0

7. Bergh P, Kindblom LG, Gunterberg B, et al. Prognostic factors in chordoma of the sacrum and mobile spine: a study of 39 patients. Cancer. 2000;88:2122-2134. doi: 10.1002/(sici)10970142(20000501)88:9<2122::aid-cncr19>3.0.co;2-1

8. Boriani S, Bandiera S, Biagini R, et al. Chordoma of the mobile spine: fifty years of experience. Spine. 2006;31:493-503. doi: 10.1097/01.brs.0000200038.30869.27

9. Hanna SA, Aston WJ, Briggs TW, et al. Sacral chordoma: can local recurrence after sacrectomy be predicted? Clin Orthop Relat Res. 2008;466:2217-2223. doi: 10.1007/s11999-008-0356-7

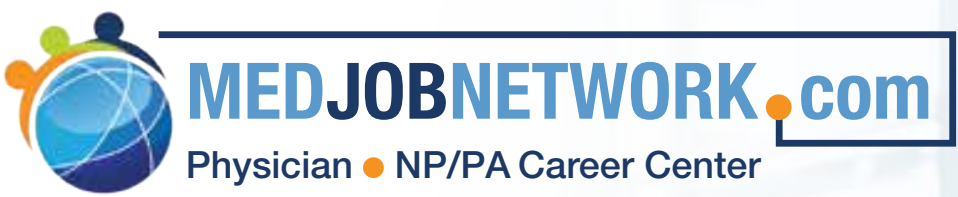

The first mobile job board for Physicians, NPs, and PAs Mobile Job Searches-access MedJobNetwork.com
on the go from your smartphone or tablet

Advanced Search Capabilities - search for jobs by specialty, job title, geographic location, employer, and more

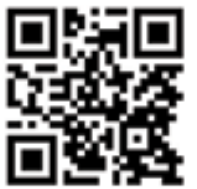

Scan this $Q R$ code

to access the mobile version of MedJobNetwork.com 